\title{
Insulin Resistance in Diabetic CKD: A Hospital-Based Study in Egypt
}

\author{
Heba A. Attea ${ }^{1^{*}}$, Alaa El Din S. Abd El Hamid², Mohammed M. Keshawy', \\ Mohamed S. Khedr'
}

${ }^{1}$ Department of Internal Medicine, Faculty of Medicine, Suez Canal University; ${ }^{2}$ Department of Clinical Pathology, Faculty of Medicine, Suez Canal University, Egypt

\begin{abstract}
Background: The presence of insulin resistance (IR) and compensatory hyper-insulinemia is strongly associated with chronic kidney disease (CKD) stages and constellates with other abnormalities such as obesity, hypertension, and dyslipidemia. CKD is associated with significant morbidity and mortality. A variety of factors, including inflammation, oxidative stress and metabolic acidosis are associated with insulin resistance. Aim: To identify the relationship between insulin resistance and diabetic kidney disease (DKD). Patients and Methods: The study was performed as a cross-sectional study including 84 patients with type 2 diabetes mellitus admitted in Suez Canal University Hospitals. Results: In diabetics with normal kidney function $28.6 \%$ of had IR, while $78.6 \%$ of diabetic patients with late DKD had IR according to HOMA- IR with significant relationship between the progress of DKD and IR ( $p$ value $\leq 0.05$ ). Conclusion: The presence of insulin resistance is strongly associated with DKD and its progress.
\end{abstract}

Keywords: Diabetes, dyslipidemia, kidney

\section{Introduction}

Diabetes mellitus is considered as one of the highly prevalent diseases. Diabetes is 1 of 4 priority non-communicable diseases targeted by world leaders and all health organizations $^{(1)}$. Both types of diabetes can lead to CKD and eventually end stage renal disease (ESRD). But because of the much higher prevalence of type 2 diabetes mellitus (T2DM) than type 1 diabetes mellitus (T1DM), often patients with ESRD have $\mathrm{T}_{2} \mathrm{DM}^{(2)}$. Patients who have insulin resistance (IR) are at enhanced risk of early onset of albuminuria and display accelerated progression of albuminuria when compared with T1DM subjects of similar age and adult T2DM patients with similar duration of diabetes(3). The severity of IR also strongly relates with the development of microalbuminuria in patients with T2DM and normal renal function ${ }^{(4)}$.

\section{Patients and Methods}

The study was performed as a cross- sectional study including 84 patients with T2DM admitted in Nephrology unit at Suez Canal University Hospitals from 2018 to 2020. They were divided into 3 groups: control group (diabetics with normal kidney function), the case groups are of diabetics with early diabetic kidney disease (DKD) (eGFR $\geq 60 \mathrm{ml} / \mathrm{min}$ ), and diabetics with late 
DKD (eGFR $<60 \mathrm{ml} / \mathrm{min})$. Both sexes and adult patients were included in the study. Exclusion criteria include hypertension, T1DM and usage of drugs as insulin or insulin sensitizers or vitamin $D$ analogues. Chronic kidney disease is defined according to the K/DOQI definitions(5). Serum creatinine was determined by routine techniques using an automated analyzer (COBAS 6000 Automated Chemistry Analyzer), used in SCU clinical pathology laboratory and creatinine clearance was calculated using the Chronic Kidney Disease Epidemiology Collaboration equation ${ }^{(6)}$. Consistent with American Diabetes Association guidelines, albumin and creatinine was measured in a random spot urine sample. These measurements were used to calculate the urinary albumin-to-creatinine ratio. Diabetic nephropathy was defined as a urinary albumin-to-creatinine ratio $\geq 30 \mathrm{mg} / \mathrm{g}(7)$. Lipid profile including serum total cholesterol, triglyceride, low density lipoprotein (LDL) and high-density lipoprotein (HDL). Insulin resistance was assessed by the homoeostasis model (HOMA). The HOMA test estimated IR from fasting glucose and insulin concentrations. The insulin level was measured by mean of competitive enzyme immunoassay with a double antibody procedure using Insulin ELISA Kit BDIN31BA (96 Tests), Germany. Patient with IR defined by HOMA-IR $>2^{(8)}$. The collected data was coded, and the statistical analysis done using the SPSS (statistical package for social sciences) version 24.

\section{Results}

The socio-demographic data of the three studied groups showed that diabetics with normal kidney function; $32.1 \%$ were males and $67.9 \%$ were females with a mean age of 53.71 years, while diabetics with early DKD $39.3 \%$ were males and $60.7 \%$ were females, with a mean age of 49.93 years. Diabetics with late DKD were $35.7 \%$ males and $64.3 \%$ were females with a mean age of 56.6 years. The results showed that $28.6 \%$ of diabetics with normal kidney function had IR, while $78.6 \%$ of diabetic patients with late DKD had IR according to HOMA- IR with significant relationship between the progress of DKD and IR $(p \leq 0.05)$ (table 1$)$.

Table 1: Comparison between the three studied groups according to HOMA IR

\begin{tabular}{|c|c|c|c|c|c|c|c|c|}
\hline \multirow[t]{2}{*}{ HOMA IR } & \multicolumn{2}{|c|}{$\begin{array}{l}\text { patients with } \\
\text { early DKD } \\
(n=28)\end{array}$} & \multicolumn{2}{|c|}{$\begin{array}{l}\text { patients with } \\
\text { late DKD } \\
(n=28)\end{array}$} & \multicolumn{2}{|c|}{$\begin{array}{l}\text { patients with nor- } \\
\text { mal kidney func- } \\
\text { tion }(n=28)\end{array}$} & \multirow[t]{2}{*}{$\begin{array}{l}\text { Test of } \\
\text { Sig. }\end{array}$} & \multirow[t]{2}{*}{$\mathrm{p}$} \\
\hline & No. & $\%$ & No. & $\%$ & No. & $\%$ & & \\
\hline Normal $($ IS <2) & 17 & 60.7 & 6 & 21.4 & 20 & 71.4 & \multirow{2}{*}{$\chi^{2}=15.5^{*}$} & \multirow{2}{*}{$0.001^{*}$} \\
\hline Abnormal $(I R \geq 2)$ & 11 & 39.3 & 22 & 78.6 & 8 & 28.6 & & \\
\hline Range & \multicolumn{2}{|c|}{$0.10-27.0$} & \multicolumn{2}{|c|}{$0.26-24.0$} & \multicolumn{2}{|c|}{$0.07-15.0$} & \multirow{2}{*}{$H=4.608$} & \multirow{2}{*}{0.021} \\
\hline Mean \pm SD & \multicolumn{2}{|c|}{$3.43 \pm 4.91$} & \multicolumn{2}{|c|}{$5.18 \pm 5.21$} & \multicolumn{2}{|c|}{$2.08 \pm 4.71$} & & \\
\hline
\end{tabular}

Table (2) showed that $100 \%$ of diabetics with normal kidney function with normal HOMA-IR had normal lipid profile, while $100 \%$ of those with abnormal HOMA-IR were dyslipidemic. In diabetics with early DKD $64.7 \%$ of patients with normal HOMAIR had normal lipid profile, while $81.8 \%$ of those with abnormal HOMA-IR were dyslipidemic. In diabetics with late DKD100
$\%$ of patients with normal HOMA-IR had normal lipid profile, while $90 \%$ of those with abnormal HOMA-IR were dyslipidemic. This shows strong statistically significant relationship between dyslipidemia and insulin resistance. Lipid profile of the study participants as shown in table (3) shows that IR and DKD is directly correlated with hypertriglyceridemia and low HDL levels. 
Table (3) shows that $28.6 \%$ of patients in control group had high risk levels of HDL, while $39.3 \%$ of patients in case group (2) had high risk levels. Also, $64.3 \%$ of control group had normal triglyceride level while patients with hyper- triglyceridemia in case group (1) and case group (2) represent $28.6 \%, 64.3 \%$ respectively $(p=0.05)$.

Table 2: Relation between HOMA IR and dyslipidemia in each group

\begin{tabular}{|c|c|c|c|c|c|c|}
\hline \multirow{3}{*}{ Dyslipidemia } & \multicolumn{4}{|c|}{ HOMA IR } & \multirow{3}{*}{$\chi^{2}$} & \multirow{3}{*}{${ }^{\mathrm{FE}} \mathrm{p}$} \\
\hline & \multicolumn{2}{|c|}{$\begin{array}{l}\text { Normal } \\
(n=43)\end{array}$} & \multicolumn{2}{|c|}{$\begin{array}{c}\text { Abnormal } \\
(n=41)\end{array}$} & & \\
\hline & No. & $\%$ & No. & $\%$ & & \\
\hline $\begin{array}{l}\text { Diabetics with normal kidney func- } \\
\text { tion }(\mathrm{n}=28) \\
\text { Normal } \\
\text { Dyslipidemia }\end{array}$ & $\begin{array}{c}20 \\
0\end{array}$ & $\begin{array}{c}100.0 \\
0.0\end{array}$ & $\begin{array}{l}0 \\
8\end{array}$ & $\begin{array}{c}0.0 \\
100.0\end{array}$ & 28 & $<0.001$ \\
\hline $\begin{array}{l}\text { Diabetics with early DKD }(\mathrm{n}=28) \\
\text { Normal } \\
\text { Dyslipidemia }\end{array}$ & $\begin{array}{l}11 \\
6 \\
\end{array}$ & $\begin{array}{l}64.7 \\
35.3\end{array}$ & $\begin{array}{l}2 \\
9 \\
\end{array}$ & $\begin{array}{l}18.2 \\
81.8 \\
\end{array}$ & $4 \cdot 3$ & 0.04 \\
\hline $\begin{array}{l}\text { Diabetics with late DKD }(\mathrm{n}=28) \\
\text { Normal } \\
\text { Dyslipidemia }\end{array}$ & $\begin{array}{l}6 \\
0\end{array}$ & $\begin{array}{c}100.0 \\
0.0\end{array}$ & $\begin{array}{c}2 \\
20\end{array}$ & $\begin{array}{c}9.1 \\
90.9\end{array}$ & $14 \cdot 9$ & $<0.001$ \\
\hline
\end{tabular}

$\chi^{2}$ : Chi square; FE: Fisher Exact; p: for association between HOMA IR and dyslipidemia in each group

\section{Discussion}

The current diabetes pandemic has emerged as a global health burden. Despite of accumulating evidence supporting the prevention of obesity and related metabolic disorders, the number of diabetic patients is rapidly increasing, particularly in middle- and low-income countries ${ }^{(9)}$. According to the estimates of the International Diabetes Federation, diabetes affects 425 million people globally, and the number is expected to increase to more than 600 million in $2045^{(10)}$. It is a major concern that diabetes is associated with the development of micro and macro-vascular complications. DKD is the leading cause of end-stage renal disease and is therefore a critical issue for healthcare systems ${ }^{(7)}$. In the current study, according to HOMA- IR, $28.6 \%, 39.3 \%$, $78.6 \%$ of diabetic patients with normal kidney function, diabetic patients with early DKD and diabetic patients with late DKD respectively had IR with significant relationship between the progress of DKD and IR. These results are in accordance with these observations, and it supports the notion that kidney disease is associated with insulin resistance, hyperinsulinemia and hyperglycemia, and that insulin resistance might be an important factor in the cause and progress of CKD. Kornélia Štefíková and her collegues in 2012 demonstrated that CKD is accompanied by insulin resistance even in stages 2 and 3. This study identified a strong and significant relationship between IR, renal function and proteinuria(11). The current study shows that $28.6 \%$ of patients in diabetics with normal kidney function had dyslipidemia, while in diabetics with early DKD and in diabetics with late DKD $13.6 \%$, $71.4 \%$ respectively had dyslipidemia with significant relationship between the progress of DKD and dyslipidemia ( $p=0.006)$. The study shows strong statistically significant relationship between dyslipidemia and insulin resistance. These results are in agreement with a study conducted in 10.038 people with normal blood pressure 
or pre-hypertension demonstrated dys-lipidemia as strong predictor of development of type $2 \mathrm{DM}$ and $I \mathrm{R}^{(12)}$. Zheng and his colleagues in 2017 found that dyslipidemia induces insulin resistance and impaired $\beta$ cell response to insulin resistance in individuals with normal glucose tolerance. Furthermore, dyslipidemia diminishes $\beta$ cell function in subjects with impaired glucose tolerance. Also, triglyceride and HDL-C were correlated with $I \mathrm{R}^{(13)}$. Finally, there is significant relationship between the progress of DKD, IR, dyslipidemia, and obesity. Our study has some limitations being a single-center study with a relatively small number of patients.

Table 3: Comparison between the three studied groups according to Lipid profile

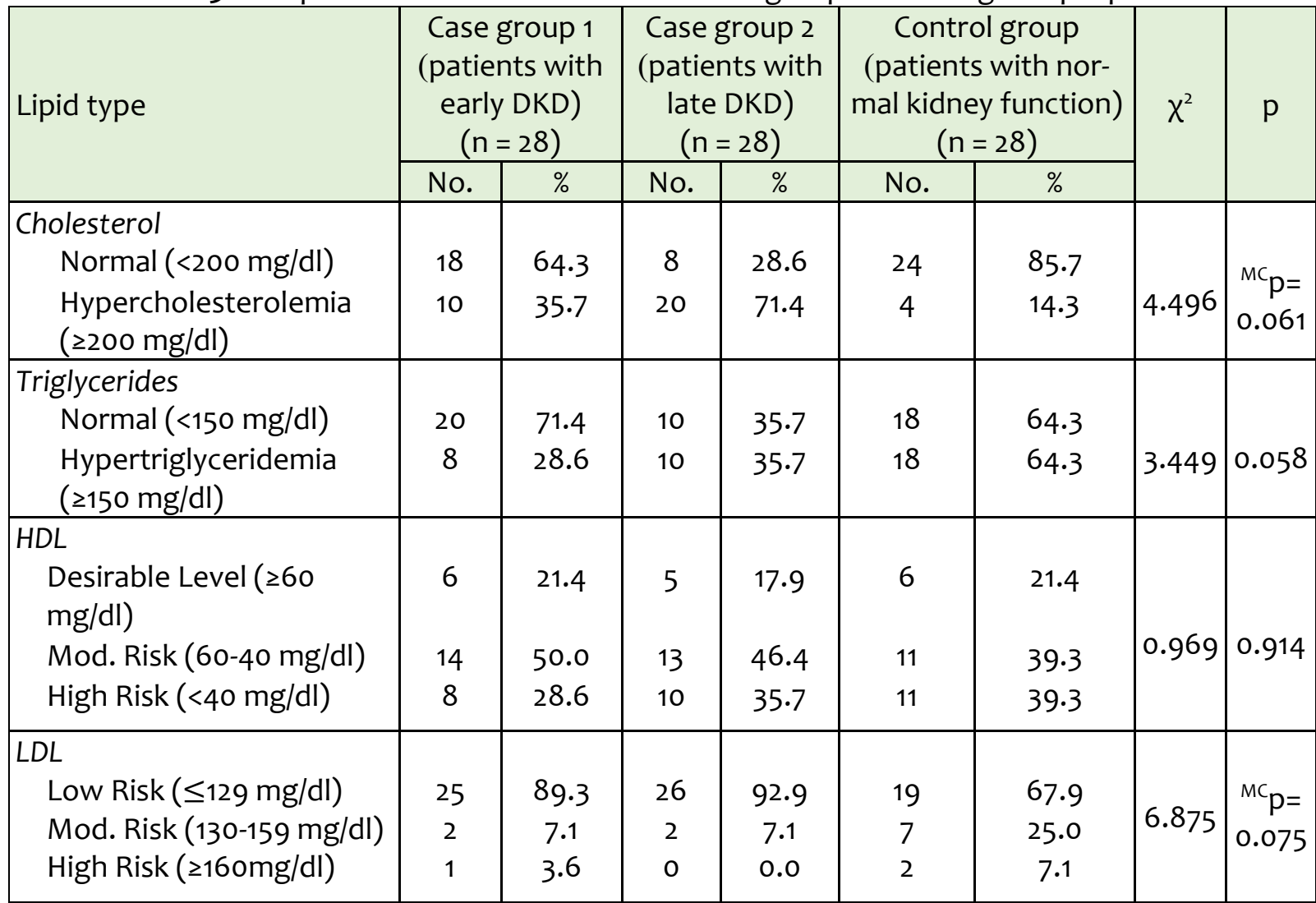

$\chi^{2}$ : Chi square test; MC: Monte Carlo; p: $p$ value for comparing between the three studied groups

\section{Conclusion}

The presence of insulin resistance and the compensatory hyper-insulinemia is strongly associated with the presence of DKD and its progress and constellates with other abnormalities such as obesity and dyslipidemia. Correction of insulin resistance in the patients of T2DM improves their resistance and their glycemic status and prevent the occurrence of complications.

\section{References}

1. GBD 2013 Risk Factors Collaborators. Global, regional, and national comparative risk assessment of 79 behavioural, environmental, and occupational, and metabolic risks or clusters of risks in 188 countries, 1990-2013: a systematic analysis for the Global Burden of Disease Study 2013. Lancet. 2015; 386(10010):2287-323. 
2. Shahbazian H and Rezaii I. (2013). Diabetic kidney disease; review of the current knowledge. J Renal Inj Prev.; 2(2): 73-80.

3. Solis-Herrera C, Triplitt CL, Lynch JL. (2014). Nephropathy in youth and young adults with type 2 diabetes. Curr Diab Rep; 14: 456.

4. Parvanova Al, Trevisan R, Iliev IP, et al. (2006). Insulin resistance and microalbuminuria: a cross-sectional, case-control study of 158 patients with type 2 diabetes and different degrees of urinary albumin excretion. Diabetes; 55: 14561462.

5. National Kidney Foundation Kidney Disease Outcome Quality Initiative (K/DOQI) Advisory Board. K/DOQI clinical practice guidelines for chronic kidney disease. Am J Kidney Dis 2002; 39 [Suppl 2]: S32-S33.

6. Levey AS., Stevens LA., Schmid CH., et al. (2009). A new equation to estimate glomerular filtration rate. Ann Intern Med. 150(9), 604-612.

7. American Diabetes Association. (2020). Microvascular Complications and Foot Care: Standards of Medical
Care in Diabetes-2020. Diabetes Care, 43, S135-S151.

8. Wallace TM, Levy JC, Matthews DR. (2004). Use and abuse of HOMA modelling. Diabetes Care. 27 (6): 1487-95.

9. International Diabetes Federation (IDF) website, 2020. http://www. idf.org/membership/mena/egypt.

10. Huang Y, Karuranga S, Malanda B., Williams D. (2018). Call for data contribution to the IDF Diabetes Atlas $9^{\text {th }}$ Edition 2019. Diabetes Res. Clin. Pract., 140, 351-352.

11. Stefikova K, Spustova V, Krivosikova Z. (2011). Insulin Resistance and Vitamin D Deficiency in Patients with Chronic Kidney Disease Stage 2-3. Physiol. Res. 60: 149-155.

12. Ginsberg HN, Zhang YL, HernandezOno A. (2006). Metabolic Syndrome focus on dyslipidemia. Obesity. 14 (suppl1): 41s-9s.

13. Zheng S, Xu H, Zhou H, et al. (2017). Associations of lipid profiles with insulin resistance and $\beta$ cell function in adults with normal glucose tolerance and different categories of impaired glucose regulation. PLoS ONE 12(2): e0172221. 